\title{
Intricate Interplay of Entwined Metabolic and Inflammatory Life-threatening Processes in Tumor Lysis Syndrome Complicating Prostate Cancer: A Systematic Review with a Single Institution Experience
}

\author{
Dawood Findakly ${ }^{1}$, Jue Wang ${ }^{2}$ \\ 1. Internal Medicine, Creighton University Arizona Health Education Alliance / Valleywise Health Medical Center \\ (formerly MIHS), Phoenix, USA 2. Genitourinary Oncology, Creighton University School of Medicine / University of \\ Arizona Cancer Center at Dignity Health St. Joseph's, Phoenix, USA
}

Corresponding author: Jue Wang, jue.wang@dignityhealth.org

\begin{abstract}
Tumor lysis syndrome (TLS) occurs in rapidly proliferating tumor cells, either spontaneously or after cytotoxic therapy. It has been well-documented in hematological diseases but is extremely rare in solid neoplasms, particularly in prostate cancer (PRCA). In the presence of risk factors, it can cause metabolic disturbances and be potentially fatal.
\end{abstract}

\begin{abstract}
We searched PubMed, Medline, ScienceDirect, and Scopus for "tumor lysis syndrome" and "prostate cancer" and conducted a systematic review with a pooled analysis for the published literature and cases from our institution. Twenty-two TLS cases were identified (18 published in the literature and four cases from our institution). The patients' median age was 68 years (range 16-82), and most cases were prostate adenocarcinoma. The median prostate-specific antigen (PSA) was 374 (range 66.7-10,867). Ten cases (45.5\%) had spontaneous TLS (STLS) while 12 cases (54.5\%) were treatment-related (TTLS). All patients had elevated lactate dehydrogenase (LDH) with other biochemical variables, and all underwent aggressive supportive therapy. Eleven patients underwent hemodialysis, 12 patients received rasburicase, while three patients received allopurinol. The mortality rate was $75 \%$ among 12 cases of TTLS, and it was $30 \%$ of the 10 cases with STLS.
\end{abstract}

Among patients with PRCA, both TTLS and STLS linked to very high mortality. Early identification of TLS would substantially attain improved survival outcomes. Hence, physicians should consider TLS as a differential diagnosis when evaluating AKI and electrolyte abnormalities, particularly in patients with metastatic PRCA and high disease burden, even before the initiation of cytotoxic therapy.

Received 03/16/2020

Review began 03/18/2020 Review ended 03/21/2020 Published 03/24/2020

() Copyright 2020

Findakly et al. This is an open access article distributed under the terms of the Creative Commons Attribution License CC-BY 4.0., which permits unrestricted use, distribution, and reproduction in any medium, provided the original author and source are credited.
Categories: Genetics, Internal Medicine, Oncology

Keywords: tumor lysis syndrome (tls), prostate cancer, metastasis, oncologic emergency, acute kidney injury (aki), cytokine storm, tumor necrosis factor-alpha, spontaneous tls (stls), treatment-related (ttls), systematic review

\section{Introduction And Background}

Tumor lysis syndrome (TLS) is a major, life-threatening oncological emergency where hasty damage of tumor cells ends in a constellation of critical metabolic derangements. These constitute hyperuricemia, hyperphosphatemia, hyperkalemia, and hypocalcemia, leading to an acute kidney injury (AKI), which further worsens TLS metabolic abnormalities and outcomes [1-5]. TLS divided into spontaneous TLS (STLS) and treatment-related (TTLS). Although TLS is widely known to occur in patients with rapidly proliferating chemosensitive hematologic diseases, it can seldom happen in solid tumors. Recent papers were published indicating prostate cancer (PRCA) as being one of the solid tumors that can exceptionally rarely be complicated by TLS [6-24]. This study's objective is to investigate the clinical characteristics, management, and outcomes of TLS, a rare oncologic emergency, in patients with PRCA.

\section{Review}

\section{Aim of the study}

This review aims to examine the available published information on the clinical characteristics, treatment, and outcomes of TLS in patients with prostate cancer.

\section{Patients and methods}

Literature Search Strategy 
A systematic analysis through a comprehensive literature search was conducted and pooled with cases from our institution. We searched PubMed, Medline, ScienceDirect, and Scopus for "tumor lysis syndrome" and "prostate cancer." The identified list of cases and abstracts were reviewed and screened for any additional articles of interest from reference lists.

Data Extraction and Statistical Analysis

We extracted data on age at diagnosis, histological subtypes, prostate-specific antigen, Gleason score, diagnostic radiology, comorbid status, treatment, and outcomes were also recorded when available. Four cases from our institution were included in our analysis. We interpreted the pooled data and summarized it through descriptive statistics using central tendency and dispersion measures.

\section{Results}

We recognized 18 publications where TLS occurred in the setting of PRCA (14 case reports and four meeting abstracts). Out of the 18 cases of TLS, we identified 12 TTLS cases and six STLS cases. Furthermore, we identified four cases of STLS diagnosed in our institutional database. The final cohort was consistent with a total of 22 PRCA patients with TLS.

The demographics, clinicopathologic features, and survival outcomes of 22 cases of TLS in PRCA are summarized in Table 1 . The mean age $( \pm \mathrm{SD})$ was $63.5( \pm 14.5)$ years. The median prostate-specific antigen (PSA) was 374 (range 66.7-10,867 ng/mL). Most cases were prostate adenocarcinoma (except prostate rhabdomyosarcoma in one case, prostate small cell carcinoma (SCC) in two cases, and pathology type not documented in four cases). High-grade Gleason 8+ PRCA documented in 80\% of cases with available data, including two cases of prostate SCC. All cases (100\%) reported distant metastases. Of the cases with available data, $100 \%$ had bone metastasis and $52 \%$ had liver metastasis. Ten cases (45.5\%) had STLS. In contrast, 12 TLS cases (54.5\%) were associated with a variety of treatment regimens before developing TLS; out of these, six patients received chemotherapy (27.3\%), three cases received hormonal treatment (13.6\%), two received radiation therapy (9.1\%), and one case developed TLS after treatment with methylprednisolone for pembrolizumab-induced acute liver injury (4.5\%). All patients had elevated lactate dehydrogenase (LDH) with other biochemical variables such as uric acid, potassium, phosphorus, and creatinine. All patients received aggressive supportive therapy, 11 (50\%) underwent hemodialysis, 12 (54.5\%) received rasburicase, and three (13.6\%) received allopurinol (Figure 1). 


\section{Cureus}

\begin{tabular}{|c|c|c|c|c|c|c|c|c|c|c|c|c|}
\hline Author & Year & Age & Histology & $\begin{array}{l}\text { Gleason } \\
\text { score }\end{array}$ & PSA & Mets & Treatment preceding TLS & $\begin{array}{l}\text { Time to } \\
\text { TLS (days) }\end{array}$ & Rasburicase & Other management & Outcome & Ref \\
\hline Sorscher & 2004 & 80 & Adenocarcinoma & $3+3$ & 348 & Bone & Docetaxel, dexamethasone & 1 & No & Furosemide & Died & [6] \\
\hline $\begin{array}{l}\text { Tanvetyanon and } \\
\text { Choudhury }\end{array}$ & 2004 & 77 & Adenocarcinoma & ND & 10,867 & Bone, liver & Flutamide, goserelin & 6 & No & Supportive therapy & Died & [7] \\
\hline Wright et al. & 2005 & 60 & Adenocarcinoma & $3+4$ & 5520 & Bone & Paclitaxel & 1 & No & Hemodialysis & Died & [8] \\
\hline Lin et al. & 2007 & 72 & Adenocarcinoma & ND & 66.7 & Bone, liver & $\begin{array}{l}\text { Flutamide, leuprolide, } \\
\text { dexamethasone, } \\
\text { medroxyprogesterone }\end{array}$ & 21 & No & $\begin{array}{l}\text { Hemodialysis, } \\
\text { furosemide, allopurinol }\end{array}$ & Died & [9] \\
\hline Hashem et al. & 2010 & 73 & Adenocarcinoma & ND & ND & Bone & STLS & ND & No & Hemodialysis & Died & [10] \\
\hline Zulqarnain et al. & 2012 & 56 & Prostate SCC & ND & 6.1 & $\begin{array}{l}\text { Bone, LN, liver, } \\
\text { lungs }\end{array}$ & Chemotherapy & 2 & Yes & $\begin{array}{l}\text { Exchange transfusion for } \\
\text { methemoglobinemia }\end{array}$ & Alive & [11] \\
\hline Kaplan et al. & 2012 & 60 & Adenocarcinoma & $5+4$ & 300 & Bone & Radiation therapy to shoulder & 6 & Yes & Sodium bicarbonate & Died & [12] \\
\hline $\begin{array}{l}\text { Nguyen and } \\
\text { Ticona }\end{array}$ & 2014 & 72 & Adenocarcinoma & ND & ND & Bone, LN & STLS & ND & Yes & Supportive therapy & Alive & [13] \\
\hline $\begin{array}{l}\text { Watanabe and } \\
\text { Tanaka }\end{array}$ & 2014 & 16 & $\begin{array}{l}\text { Prostate } \\
\text { rhabdomyosarcoma }\end{array}$ & ND & ND & Bone, LN & STLS & ND & Yes & Supportive therapy & Alive & [14] \\
\hline Mazzoni & 2016 & 62 & Adenocarcinoma & ND & ND & Bone, LN, bladder & $\begin{array}{l}\text { Radiation, TURBT, leuprolide, } \\
\text { bicalutamide }\end{array}$ & ND & Yes & $\begin{array}{l}\text { Hemodialysis, sodium } \\
\text { bicarbonate }\end{array}$ & Died & [15] \\
\hline $\begin{array}{l}\text { Serling-Boyd et } \\
\text { al. }\end{array}$ & 2017 & 56 & Adenocarcinoma & $5+4$ & 548 & LN, liver & STLS & ND & Yes & $\begin{array}{l}\text { Sodium bicarbonate, } \\
\text { furosemide, allopurinol }\end{array}$ & Died & [16] \\
\hline $\begin{array}{l}\text { Ignaszewski and } \\
\text { Kohlitz }\end{array}$ & 2017 & 69 & Adenocarcinoma & ND & ND & Bone, liver & STLS & ND & Yes & $\begin{array}{l}\text { Hemodialysis, sodium } \\
\text { bicarbonate }\end{array}$ & Died & [17] \\
\hline $\begin{array}{l}\text { Bnarawal and } \\
\text { Varma }\end{array}$ & 2017 & 67 & ND & ND & 4500 & Bone, $L N$, liver & Docetaxel & 3 & No & Supportive therapy & Died & [18] \\
\hline Gongora et al. & 2018 & 46 & Adenocarcinoma & $4+4$ & 917 & $\begin{array}{l}\text { Bone, } \mathrm{LN} \text {, liver, } \\
\text { lungs }\end{array}$ & Carboplatin, etoposide & 5 & Yes & Supportive therapy & Alive & [19] \\
\hline McGhee-Jez et al. & 2018 & 49 & Adenocarcinoma & ND & 24.9 & Bone & STLS & ND & No & $\begin{array}{l}\text { Hemodialysis, } \\
\text { allopurinol, prednisone }\end{array}$ & Alive & [20] \\
\hline Oshima et al. & 2019 & 77 & ND & $5+4$ & $>1000$ & Bone, LN, liver & Cabazitaxel & 3 & Yes & $\begin{array}{l}\text { Hemodialysis, sodium } \\
\text { bicarbonate }\end{array}$ & Died & [21] \\
\hline Sharma and Lane & 2019 & 59 & ND & ND & 2.1 & $\begin{array}{l}\mathrm{LN} \text {, liver, adrenal } \\
\text { glands }\end{array}$ & Enzalutamide & 30 & Yes & Hemodialysis & Died & [22] \\
\hline $\begin{array}{l}\text { Mayer and } \\
\text { Zarouk }\end{array}$ & 2019 & 71 & ND & ND & ND & $\begin{array}{l}\text { ND on metastatic } \\
\text { sites }\end{array}$ & $\begin{array}{l}\text { Methylprednisolone for } \\
\text { pembrolizumab-induced acute liver } \\
\text { injury }\end{array}$ & 3 & Yes & Hemodialysis & Alive & [23] \\
\hline Case 1, Wang & 2016 & 72 & Adenocarcinoma & $4+5$ & 746 & Bone, LN & STLS & ND & No & Supportive therapy & Alive & [24] \\
\hline Case 2, Wang & 2018 & 53 & Adenocarcinoma & $4+4$ & 374 & Bone, $L N$, lungs & STLS & ND & No & Hemodialysis & Alive & [24] \\
\hline Case 3 & 2018 & 82 & Adenocarcinoma & ND & ND & Bone, LN, liver & STLS & ND & No & Supportive therapy & Alive & [24] \\
\hline Case 4 & 2019 & 69 & Prostate SCC & ND & ND & $\begin{array}{l}\text { Bone, LN, liver, } \\
\text { lungs, brain, adrenal }\end{array}$ & STLS & ND & Yes & Hemodialysis & Alive & [24] \\
\hline
\end{tabular}

TABLE 1: Summary of available publications and our hospital data for characteristics of patients with TLS in PRCA

PRCA: prostate cancer; TLS: tumor lysis syndrome; PSA: prostate-specific antigen; Mets: metastases locations; Ref: references; ND: no data; STLS: spontaneous TLS; SCC: small cell carcinoma; LN: Iymph nodes; TURBT: transurethral resection of bladder tumor 


\section{Cureus}

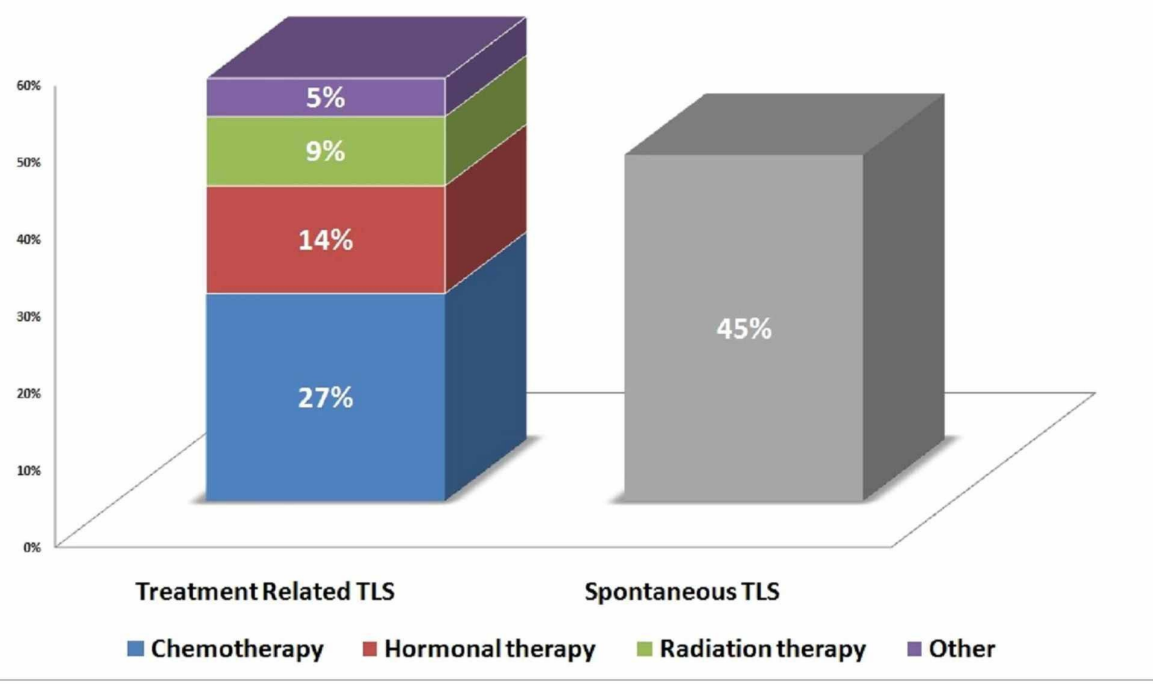

FIGURE 1: Incidence rates of treatment-related versus spontaneous TLS in PRCA patients

TLS: tumor lysis syndrome; PRCA: prostate cancer.

The mortality rate was $75 \%$ among 12 cases of TTLS, with a median survival of 4.5 days (range 1-30) from diagnosis of TLS to death, and $30 \%$ in the 10 cases with STLS. There was no reported mortality in the four cases recognized and managed in our institution.

\section{Discussion}

In our cohort, several findings are noteworthy. First, the high mortality in TLS most often occurs in PRCA patients with advanced-stage metastatic disease, significant disease burden, high Gleason score, and aggressive histology. Second, TLS in PRCA, particularly TTLS, displays poorer prognosis when compared to hematologic malignancies [25-27]. Third, we recognized two patients with primary prostate SCC. Similar to SCC in other locations, prostatic SCC histology has been linked to TLS and is known for its aggressive biological behavior and rapid proliferation rate [28-29]. Molecular studies identified a germline breast cancer susceptibility 2 (BRCA2) gene mutation in both cases. BRCA-positive PRCA patients tend to have a higher Gleason score, higher PSA, higher grade of tumor proliferation, and higher rate of metastasis, predisposing them to an early-onset, aggressive, and potentially fatal disease as compared to patients with BRCA negative tumors [30-33].

Research in animal models proposed additional mechanisms that might explain the higher mortality of TLS. Studies in mice found disseminated microemboli from lysed tumor cells during the histopathological postmortem examination; these result in widespread tissue damage, multiorgan failure, and death [34-35]. Those findings closely mimic the human autopsy findings of disseminated tumor embolism leading to massive tissue organ necrosis and death [36-37]. Furthermore, concurrent disseminated intravascular coagulation was proposed as a probable mechanism that could synergistically lead to increased mortality in patients with TLS through common pathogenesis and pro-inflammatory cytokine-mediated processes [1314]. Further, upon investigating this entity, cytokines, especially TNF-alfa (TNF $\alpha$ ) and Interferon-gamma (IFNY), are considered key mediators of what is known as a "cytokine storm," first described by Aikawa in 1996 [38]. The cytokine storm reported having a positive correlation with the tumor burden and was thought to play a vital role in the pathophysiology of TLS [39-41]. A cohort by Nakamura et al. found an elevated serum interleukin-6 (IL-6), IL-8, and IL-10 cytokine levels in all treatment-related TLS patients and reported a $100 \%$ survival rate after receiving continuous hemodiafiltration for cytokine removal [41].

We were able to identify four cases of TLS in PRCA patients managed in our 450+ bed hospital, two of which were in the last 24 months. Since our last publication on this matter, we continued to have improved outcomes through our continued efforts for early identification of TLS in patients with PRCA [24,27].

\section{Limitations of the study}

Due to the inherent nature of retrospective studies, we were not able to perform a comprehensive assessment of factors that may impact the prognosis of TLS in prostate cancer patients, including the 
performance status and comorbid conditions. Larger sample size would further contribute to the stratification of the risk of patients based on treatment type before TLS development. Despite the limitations, this systematic review presents the most updated real-world insight regarding the diagnosis and prognosis of TLS in patients with metastatic prostate cancer. Our study will contribute to the evolving understanding of TLS in solid neoplasms and have implications for future cancer treatment paradigms in the era of targeted therapy.

\section{Conclusions}

Our study underscores that TLS is a real oncologic emergency, especially among patients with advanced PRCA. A heightened state of readiness orchestrated with increased attention to improve early detection of electrolytes abnormalities is immensely important for early TLS recognition and prompt intervention in patients with newly diagnosed metastatic PRCA even before starting cancer therapy. Further research is needed to determine the true incidence and molecular markers for TLS in solid tumors, which could, therefore, aid in improving outcomes.

\section{Additional Information \\ Disclosures}

Conflicts of interest: In compliance with the ICMJE uniform disclosure form, all authors declare the following: Payment/services info: All authors have declared that no financial support was received from any organization for the submitted work. Financial relationships: All authors have declared that they have no financial relationships at present or within the previous three years with any organizations that might have an interest in the submitted work. Other relationships: All authors have declared that there are no other relationships or activities that could appear to have influenced the submitted work.

\section{References}

1. Abu-Alfa AK, Younes A: Tumor lysis syndrome and acute kidney injury: evaluation, prevention, and management. Am J Kidney Dis. 2010, 55:S1-S13. 10.1053/j.ajkd.2009.10.056

2. Howard SC, Jones DP, Pui CH: The tumor lysis syndrome. N Engl J Med. 2011, 364:1844-1854. 10.1056/NEJMra0904569

3. Darmon M, Vincent F, Camous L, et al.: Tumour lysis syndrome and acute kidney injury in high-risk haematology patients in the rasburicase era. A prospective multicentre study from the Groupe de Recherche en Réanimation Respiratoire et Onco-Hématologique. Br J Haematol. 2013, 162:489-497. 10.1111/bjh.12415

4. Mirrakhimov AE, Ali AM, Khan M, Barbaryan A: Tumor lysis syndrome in solid tumors: an up to date review of the literature. Rare Tumors. 2014, 6:68-76. 10.4081/rt.2014.5389

5. Vaisban E, Braester A, Mosenzon O, Kolin M, Horn Y: Spontaneous tumor lysis syndrome in solid tumors: really a rare condition?. Am J Med Sci. 2003, 325:38-40. 10.1097/00000441-200301000-00008

6. Sorscher SM: Tumor lysis syndrome following docetaxel therapy for extensive metastatic prostate cancer . Cancer Chemother Pharmacol. 2004, 54:191-192. 10.1007/s00280-004-0827-9

7. Tanvetyanon T, Choudhury AM: Fatal acute tumor lysis syndrome, hepatic encephalopathy and flare phenomenon following combined androgen blockade. J Urol. 2004, 171:1627. 10.1097/01.ju.0000115884.21275.f4

8. Wright JL, Lin DW, Dewan P, Montgomery RB: Tumor lysis syndrome in a patient with metastatic, androgen independent prostate cancer. Int J Urol. 2005, 12:1012-1013. 10.1111/j.1442-2042.2005.01196.x

9. Lin CJ, Hsieh RK, Lim KH, Chen HH, Cheng YC, Wu CJ: Fatal spontaneous tumor lysis syndrome in a patient with metastatic, androgen-independent prostate cancer. South Med J. 2007, 100:916-917. 10.1097/smj.0b013e318137a665

10. Hashem AAH, Dowod TAHM, Abdelmajeed MM: Spontaneous tumor lysis syndrome: a case report and review of literature. Pak J Med Sci. 2010, 458:463.

11. Zulqarnain S, Bradley T, Demitis S: Tumor lysis syndrome in small cell cancer of prostate . Chest. 2012, 142:626A. 10.1378/chest.1388951

12. Kaplan MA, Kucukoner M, Alpagat G, Isikdogan A: Tumor lysis syndrome during radiotherapy for prostate cancer with bone and bone marrow metastases without visceral metastasis. Ann Saudi Med. 2012, 32:306308. 10.5144/0256-4947.2012.306-308

13. Nguyen R, Ticona L: Spontaneous tumor lysis syndrome - a previously unrecognized complication of untreated prostate cancer - and DIC as the initial presentation of metastatic prostate cancer. J Hosp Med. 2014, 9:542.

14. Watanabe A, Tanaka R: Successful prevention of tumor lysis syndrome using recombinant urate oxidase in patient with metastasic and bulky prostate rhabdomyosarcoma. Case Rep Clin Med. 2014, 3:18-22. 10.4236/crcm.2014.31005

15. Mazzoni S: Tumor lysis syndrome in anti-androgen-treated metastatic prostate cancer . Int Urol Nephrol. 2016, 48:1837-1838. 10.1007/s11255-016-1369-x

16. Serling-Boyd N, Quandt Z, Allaudeen N: Spontaneous tumor lysis syndrome in a patient with metastatic prostate cancer. Mol Clin Oncol. 2017, 6:589-592. 10.3892/mco.2017.1186

17. Ignaszewski M, Kohlitz P: Treatment-naïve spontaneous tumor lysis syndrome in metastatic prostate adenocarcinoma: An unusual suspect. Am J Emerg Med. 2017, 35:1384-1. 10.1016/j.ajem.2017.05.044

18. Bhardwaj S, Varma S: Rare incidence of tumor lysis syndrome in metastatic prostate cancer following treatment with docetaxel. J Oncol Pharm Pract. 2018, 24:153-155. 10.1177/1078155217694492

19. Gongora ABL, Canedo FSNA, de Melo ALA, Bezerra ROF, Asprino PF, Camargo AA, Bastos DA: Tumor lysis syndrome after platinum-based chemotherapy in castration-resistant prostate cancer with a brca2 
mutation: a case report. Clin Genitourin Cancer. 2018, 17:61-64. 10.1016/j.clgc.2018.09.001

20. Mcghee-Jez A, Batra V, Sunder T, et al.: Spontaneous tumor lysis syndrome as presenting sign of metastatic prostate cancer. Cureus. 2018, 10:e3706. 10.7759/cureus.3706

21. Oshima M, Mayumi S, Yazaki K, et al.: Tumor lysis syndrome following cabazitaxel administration for metastatic castration-resistant prostate cancer: a case report. IJU Case Reports. 2019, 2:179-182. 10.1002/iju5.12070

22. Sharma AV, Lane ML: Tumor lysis syndrome from autolysis in prostate cancer . ATS J. 2019, 199:1. 10.1164/ajrccm-conference.2019.199.1_MeetingAbstracts.A6546

23. Mayer C, Zarouk S: Steroid-induced tumor lysis syndrome in the setting of pembrolizumab use . Am J Kidney Dis. 2019, 73:705. 10.1053/j.ajkd.2019.03.242

24. Wang J: Tumor lysis syndrome associated with prostate cancer: an under-recognized oncologic emergency . J Emerg Crit Care Diagn Manag. 2018, 1:1-3.

25. Buhmeida A, Pyrhönen S, Laato M, et al.: Prognostic factors in prostate cancer. Diagn Pathol. 2006, 1:4. 10.1186/1746-1596-1-4

26. Gemici C: Tumour lysis syndrome in solid tumours . Clin Oncol. 2006, 18:773-780. 10.1016/j.clon.2006.09.005

27. Harmon J, Allen S, Stoyanova D, Ruvinov K, Cassaday J, Wang J: The clinical features, outcome and prognosis of spontaneous tumor lysis syndrome in solid tumor. J Oncol Res Forecast. 2018, 1:1009.

28. Kalemkerian GP, Darwish B, Varterasian ML: Tumor lysis syndrome in small cell carcinoma and other solid tumors. Am J Med. 1997, 103:363-367. 10.1016/s0002-9343(97)00153-8

29. Nadal R, Schweizer M, Kryvenko ON, Epstein JI, Eisenberger MA: Small cell carcinoma of the prostate . Nat Rev Urol. 2014, 11:213-219. 10.1038/nrurol.2014.21

30. Sundararajan S, Ahmed A, Goodman OB Jr: The relevance of BRCA genetics to prostate cancer pathogenesis and treatment. Clin Adv Hematol Oncol. 2011, 9:748-755.

31. Mitra A, Fisher C, Foster CS, et al.: Prostate cancer in male BRCA1 and BRCA2 mutation carriers has a more aggressive phenotype. Br J Cancer. 2008, 98:502-507.

32. Fiorentino M, Judson G, Penny K, et al.: Immunohistochemical expression of BRCA1 and lethal prostate cancer. Cancer Res. 2010, 70:3136-3139. 10.1158/0008-5472.CAN-09-4100

33. Agalliu I, Gern R, Leanza S, Burk RD: Associations of high-grade prostate cancer with BRCA1 and BRCA2 founder mutations. Clin Cancer Res. 2009, 15:1112-1120. 10.1158/1078-0432.CCR-08-1822

34. Lovelace K, van Gessel Y, Asher LV, Vogel P: Spontaneous acute tumor lysis syndrome in a DBA/1J mouse: a case report and review. Review Toxicol Pathol. 2003, 31:486-490. 10.1080/01926230390224674

35. Vogel P, Pletcher JM, Liang Y: Spontaneous acute tumor lysis syndrome as a cause of early deaths in shortterm carcinogenicity studies using p53 +/- mice. Vet Pathol. 2010, 47:719-724. 10.1177/0300985810363484

36. Takeuchi N, Miyazawa S, Ohno Z, Yoshida S, Tsukamoto T, Fujiwara M: A case of spontaneous tumor lysis syndrome in malignant melanoma. World J Oncol. 2016, 7:40-44. 10.14740/wjon970w

37. Ito T, Ohta T, Narumi M, Sakaki H, Seino M, Sudo T, Nagase S: Tumor lysis syndrome associated with docetaxel and carboplatin in a case with recurrent endometrial cancer. Gynecol Oncol Rep. 2018, 24:21-23. 10.1016/j.gore.2018.02.005

38. Aikawa N: Cytokine storm in the pathogenesis of multiple organ dysfunction syndrome associated with surgical insults [Article in Japanese]. Nihon Geka Gakkai Zasshi. 1996, 97:771-777.

39. Smith L, Venella K: Cytokine release syndrome: inpatient care for side effects of CAR T-cell therapy . Clin J Oncol Nurs. 2017, 21:29-34. 10.1188/17.CJON.S2.29-34

40. Shimabukuro-Vornhagen A, Gödel P, Subklewe M, et al.: Cytokine release syndrome. J Immunother Cancer. 2018, 6:56-10. 10.1186/s40425-018-0343-9

41. Nakamura M, Oda S, Sadahiro T, Hirayama Y, Tateishi Y, Abe R, Hirasawa H: The role of hypercytokinemia in the pathophysiology of tumor lysis syndrome (TLS) and the treatment with continuous hemodiafiltration using a polymethylmethacrylate membrane hemofilter (PMMA-CHDF). Case Reports Transfus Apher Sci. 2009, 40:41-47. 10.1016/j.transci.2008.11.004 